\title{
Repair of articular cartilage and subchondral defects in rabbit knee joints with a polyvinyl alcohol/nano- hydroxyapatite/polyamide 66 biological composite material
}

Tao Guo ${ }^{1^{*}}$, Xiaobin Tian', Bo Li', Tianfu Yang ${ }^{2}$ and Yubao Li $^{3}$

\begin{abstract}
Background: This study sought to prepare a new PVA/n-HA/PA66 composite to investigate the repair of articular cartilage and subchondral defects in rabbit knee joints.

Methods: A $5 \times 5 \times 5$ mm-sized defect was created in the patellofemoral joints of 72 healthy adult New Zealand rabbits. The rabbits were then randomly divided into three groups $(n=24)$ : PVA/n-HA+PA66 group, polyvinyl alcohol (PVA) group, and control (untreated) group. Cylindrical PVA/n-HA+PA66, $5 \times 5 \mathrm{~mm}$, comprised an upper PVA layer and a lower n-HA+PA66 layer. Macroscopic and histological evaluations were performed at 4, 8, 12, and 24 weeks, postoperatively. Type II collagen was measured by immunohistochemical staining. The implant/cartilage and bone interfaces were observed by scanning electron microscopy.

Results: At 24 weeks postoperatively, the lower PVA/n-HA+PA66 layer became surrounded by cartilage, with no obvious degeneration. In the PVA group, an enlarged space was observed between the implant and the host tissue that had undergone degeneration. In the control group, the articular cartilage had become calcified. In the PVA/n-HA+PA66 group, positive type II collagen staining was observed between the composite and the surrounding cartilage and on the implant surface. In the PVA group, positive staining was slightly increased between the PVA and the surrounding cartilage, but reduced on the PVA surface. In the control group, reduced staining was observed throughout. Scanning electron microscopy showed increased bone tissue in the lower n-HA+PA66 layer that was in close approximation with the upper PVA layer of the composite. In the PVA group, the bone tissue around the material had receded, and in the control group, the defect was filled with bone tissue, while the superior aspect of the defect was filled with disordered, fibrous tissue.
\end{abstract}

Conclusion: The diphase biological composite material PVA/n-HA+PA66 exhibits good histocompatibility and offers a satisfactory substitute for articular cartilage and subchondral bone.

Keywords: Biological composite material, Articular cartilage, Subchondral bone, Repair

\footnotetext{
* Correspondence: drguotao04b@sina.com

'Department of Orthopedics, Guizhou Province People's Hospital, Guiyang,

Guizhou province 550002, China

Full list of author information is available at the end of the article
} 


\section{Background}

Articular cartilage is a type of hyaline cartilage characterized by low friction, good elasticity, and hypertonicity that has an important role in maintaining joint movement. Trauma and osteoarthritis are the two factors that can lead to articular surface defects, which eventually develop into joint dysfunction. Joint fusion or joint replacement is necessary to repair joint dysfunction, because articular cartilage itself lacks a regenerative capacity $[1,2]$. Adult articular cartilage has a limited reparatory capability; that is, it can partially or totally repair itself when the defect diameter is less than $3 \mathrm{~mm}$ but not when the diameter exceeds $3 \mathrm{~mm}$, in which case it will develop into arthritis [3]. Joint replacement is not ideal for young and middleaged patients because the joint implant has a limited life expectancy. For this reason, an effective method for repairing articular cartilage defects is an emergent issue in the field of orthopedics.

Previous methods used to repair articular cartilage defects include enhancement of the articular cartilage healing capacity and biological repair by autologous or xenogenic tissue grafting. The latter method includes autologous periosteal, perichondrial, and osteochondral grafting as well as allogeneic osteochondral grafting. But the long-term therapeutic effects of these methods are not satisfactory [4]. With advances in tissue engineering technology, some progress has been made in the repair of articular cartilage defects. Nevertheless, repair of articular cartilage to a high standard, with satisfactory longterm therapeutic effects, has not been acquired in the clinic $[5,6]$. At present, the artificial materials used in the treatment of advanced osteoarthrosis are made of metal, ceramics, and ultra-high molecular weight polyethylene, with increasing attention being paid to the postoperative complications caused by implant abrasion and loosening. Therefore, some researchers are investigating alternative artificial cartilage substitutes as a repair strategy [7].

Elastomeric materials with a similar biomechanical property to cartilage have been mostly selected as alternative materials for cartilage replacement therapies, such as silicon rubber, polyurethane, and polyvinyl alcohol (PVA) hydrogel. Silicon rubber is no longer a popular material choice, owing to its ease of abrasion and absorption of oil in the body [8]. Polyurethane, as a long-term implant material, requires an improvement in its degradation rate. Moreover, the hydrolysate of the curing agent diisocyanate is a potential carcinogenic substance [9]. Polyvinyl alcohol (PVA) hydrogel, on the other hand, is a porous material similar to natural cartilage and is considered by some to be an ideal alternative material to cartilage [10]. It is a water-soluble synthetic polymer hydrolyzed by polyvinyl acetate that has been widely used as artificial cartilage, drug delivery systems, artificial meniscus, heart valve, anti-thrombin materials, vascular grafts, and biomedical sponges in the field of biomedicine for its high elasticity, stable chemical property, ease of molding, absence of toxicity and adverse reactions, and good histocompatibility within the human body [11-16].

Despite its suitable properties, the use of the PVA hydrogel in an articular cartilage and subchondral bone defect remains a concern for orthopedists. Previously, the implanted PVA was fixed by sticking or sutures, which produced poor fixation and led to the formation of a gap between the PVA and the subchondral bone. Fabricating the PVA biomaterial into a cylinder, which can be inserted into the articular cartilage and subchondral bone, offers poor stability to the joint. Likewise, mechanical fixation and chemical fixation have also yielded unsatisfactory outcomes $[17,18]$. Therefore, the application of PVA for the repair of articular cartilage defects is limited.

In this study, we prepared a novel artificial bone material comprising nano-hydroxyapatite (n-HA) and polyamide 66 (PA66) (n-HA+PA66) to form a base for insertion of the PVA hydrogel. This double-layered biomaterial PVA/n-HA+PA66 composite was prepared using the in situ synthesis and freeze-thaw cycle method. The upper layer of the biomaterial composite was made of PVA and used as a substitute for articular cartilage, while the lower layer was made of n-HA+PA66 and used to substitute for subchondral bone. The upper layer and lower layer were firmly connected prior to implantation in a rabbit patellofemoral defect model to assess its potential use as a therapeutic grafting substitute.

\section{Methods \\ Materials}

Biomaterial composites PVA/n-HA+PA66 and PVA were provided by the Nanometer Analytical and Testing Center, Sichuan University, China.

\section{Animals}

This study was performed at the Laboratory of Tissue Engineering, Department of Orthopedics, Huaxi Hospital, Sichuan University, China between July 2014 and July 2016. Seventy-two healthy adult New Zealand rabbits, aged 5-8 months, weighing 160-240 g, and with an equal number of males and females, were provided by the Laboratory Animal Center, Sichuan University, China (license No. SCXK (Chuan) 2004-14).

\section{Model preparation and group management}

Seventy-two rabbits (36 male and 36 female) were randomly divided into three groups with 24 rabbits in each group: PVA/n-HA+PA66 implant (contains 11 male and 13 female), PVA implant (contains 14 male and 10 female), and control (contains 11 male and 13 female). Following anesthesia by an intraperitoneal injection of $30 \mathrm{~g} / \mathrm{L}$ pentobarbital, an incision was made on the skin 
around the medial patella to expose the patellofemoral joint. The femoral bone joint was perforated (diameter $5 \mathrm{~mm}$, depth $5 \mathrm{~mm}$ ) to prepare models of articular cartilage and subchondral bone defects. The double-layer biomaterial composite PVA/n-HA+PA66 and simple PVA were implanted into the hole, respectively, and lightly pressed through the use of an inserter to stabilize the implant, the control group received no treatment. Each rabbit was intramuscularly injected with 40,000 units of penicillin per day for 3 days after surgery. After wound sutures, the operated limbs were not immobilized and allowed to move freely.

\section{Gross observation}

After surgery, the diet, activity level, wound healing, and range of motion were assessed for each rabbit. At a postoperative time period of $4,8,12$, or 24 weeks, rabbits were sacrificed under anesthesia and the articular cavity was opened to examine the cartilage surrounding the implant, synovial membrane, and implant stability and compared with the contralateral joint surface.

\section{Histomorphological observation}

Eighteen rabbits were sacrificed at postoperative 4 weeks for histomorphological observation, 18 rabbits were sacrificed at postoperative 8 weeks, and 9 rabbits were sacrificed at 12 and 24 weeks postoperatively for histomorphological observation and immunohistochemical detection. Nine rabbits were sacrificed at 12 and 24 weeks postoperatively for scanning electron microscopy observation. Following removal of condyles of the femur, a tissue specimen about $1 \mathrm{~cm}$ in length was harvested, with the implant as a center, and fixed with $40 \mathrm{~g} / \mathrm{L}$ paraformaldehyde. Specimens were then decalcified with 10\% EDTA and dehydrated and embedded with paraffin following routine procedures. Slices of $5-\mu \mathrm{m}$-thick sections were stained with hematoxylin-eosin, Masson's trichrome, and toluidine blue and observed under the microscope (BX51, Olympus, Tokyo, Japan).

\section{Immunohistochemical detection of type II collagen}

At postoperative 8,12 , and 24 weeks, the tissue specimens were harvested as described above and then fixed, decalcified, hydrated, embedded, sliced into 5- $\mu$ m-thick sections, and stained for type II collagen expression using standard immunohistochemistry. The specimens were then assessed using an inverted phase contrast microscope (BX51; Olympus).

\section{Scanning electron microscopy observation}

At postoperative 12 and 24 weeks, tissue specimens $(1 \times$ $1 \times 1 \mathrm{~cm})$ were fixed with $2.5 \%$ glutaraldehyde $(\mathrm{pH} 7.2-$ 7.4), dehydrated with ethanol series, and subject to gold sputtering. The interface between the implant and the surrounding cartilage and subchondral bone was observed by scanning electron microscopy (S3400N; Hitachi, Japan).

\section{Results}

Appearance and inner structure of biomaterial composite The cylindrical PVA/n-HA+PA66 composite was $5 \mathrm{~mm}$ in diameter and $5 \mathrm{~mm}$ in height. The upper layer of PVA was $1.5 \mathrm{~mm}$ high and consisted with an ivory, smooth, and tenacious surface. By comparison, the lower layer of $\mathrm{n}-\mathrm{HA}+\mathrm{PA} 66$ was $3.5 \mathrm{~mm}$ high, and had a rough and stiff composition. Using scanning electron microscopy, the PVA exhibited a porous structure, with a pore diameter of $5-40 \mu \mathrm{m}$, a porosity of $75.3 \%$ and water ratio of $71.6 \%$. The n-HA+PA66 component of the biomaterial was also porous, with a pore diameter of $100-400 \mu \mathrm{m}$ and porosity of $61.7 \%$. By X-ray diffraction analysis, the PVA appeared as a semi-crystalline polymer, with strong hydrogen bonds between the hydroxyl groups. The composite n-HA+PA66 consisted of a uniform crystal structure, with firm bonds to polyamide (Fig. 1).

\section{Biological behaviors of rabbits}

Seventy-two New Zealand rabbits were included in the final analysis. After surgery, no rabbits had died or suffered wound infection/secretion. The rabbits in the PVA/n-HA +PA66 implant group and PVA implant group showed a good range of motion. In the control group, joint motion was reduced in two rabbits and this symptom developed into knee joint stiffness at 8 weeks after surgery.

\section{Changes in the knee joint cavity}

In the PVA/n-HA+PA66 implant group, the articular surface was smooth. At 4 weeks, the gap surrounding the implant was filled with fibrous tissue, which secured the implant such that it could not be pulled out. At 8, 12 , and 24 weeks, the gap surrounding the implant disappeared, and the joints regained good function. No obvious cartilage degeneration was observed, and the implant bonded well with the adjacent articular cartilage and subchondral bone. As expected, the contralateral articular surface was smooth without abrasion. No hyperplastic and plump synovial membrane formations were observed (Fig. 2a). In the PVA implant group, the surface of the implant was smooth at 4 weeks, but the implant was not firmly fastened and easily prolapsed. At 8 and 12 weeks, the implant had dropped by $2.0-3.0 \mathrm{~mm}$. At 24 weeks, a small amount of degenerative change was observed in the cartilage (pale color and surface layer abrasion), and the implant was not firmly fastened into the joint (Fig. 2b). In the control group, the defect region was filled with fibrous granulation tissue at 4 weeks, which gradually enclosed the articular surface. Light colored, fibrous cartilage with a roughened surface was identified. At 24 weeks, the fibrous tissue had calcified 


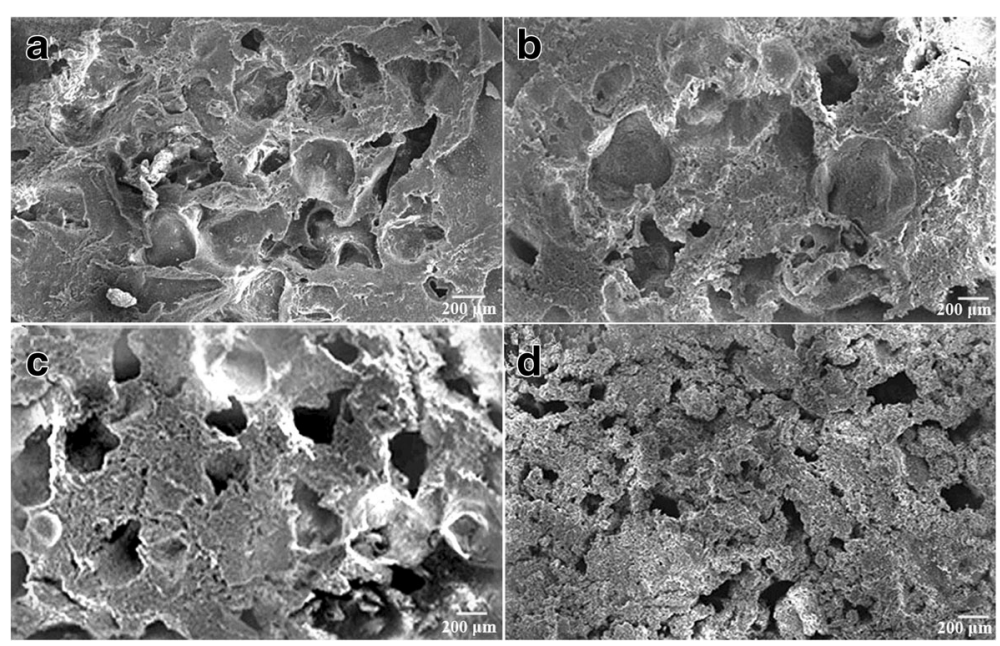

Fig. 1 Scanning electron microscopy image of the porous biomaterial nano-hydroxyapatite+polyamide $66(\times 60)(\mathbf{a} \times 80, \mathbf{b} \times 70, \mathbf{c} \times 60$, d $\times 50)$

and continued to appear rough. The articular cartilage in the surrounding region presented with degenerative changes, and the articular synovium exhibited hyperplasia, swelling, and appeared plump (Fig. 2c). Severe traumatic arthritis, articular synarthrophysis, and stiffness occurred in two of the rabbits in this group.

\section{Histomorphological observations}

At 4 weeks, for rabbits in the PVA/n-HA+PA66 implant group, the fibrous tissue in the gap between the implant and adjacent tissue contained a small number of filtrated inflammatory cells, with some fibrous tissue growing into the pores of the n-HA+PA66 biomaterial. At 8 and 12 weeks, a large amount of fibrous tissues had infiltrated into the pores of the n-HA +PA66 lower layer, with the formation of some collagen and osteoid. The gap between the PVA in the upper layer and the adjacent cartilage had become filled with articular cartilage that had also started to cover the PVA surface. At 24 weeks, the lower layer

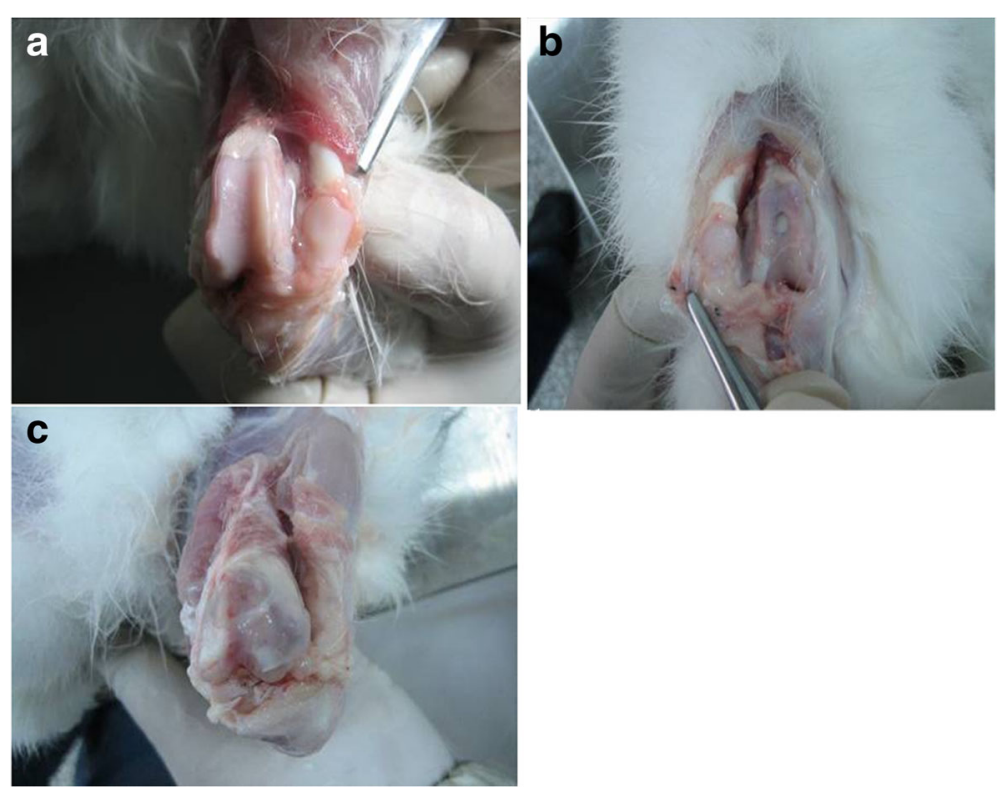

Fig. 2 At 24 weeks after implantation of polyvinyl alcohol/nano-hydroxyapatite and polyamide 66. No obvious changes were observed in the peripheral articular cartilage; the contralateral articular surface was smooth (a). At 24 weeks after implantation of polyvinyl alcohol, the implant had dropped and was not firmly fastened into the joint (b). At 24 weeks in the control group, the surface of joint appears rough and articular cartilage in the surrounding region presented with degenerative changes (c) 
had bonded with the adjacent tissue, with much osteoid in the pores that had formed a reticular layer. No abrasion to the PVA in the upper layer was observed, and the adjacent cartilage was ordered, covering the surface of the PVA, with no obvious degeneration (Fig. 3a).

In the PVA implant group, there was a large number of infiltrated inflammatory cells at 4 weeks in the gap between the implant and the adjacent subchondral bone, with some proliferative fibrous tissue. At 8 and 12 weeks, the gap between the implant and the host tissue was filled with fibrous tissue. The biomaterial adjacent to the articular cartilage edge appeared blunted and presented with slight degenerative changes. At 24 weeks, the adjacent cartilage had thinned and continued to show degenerative changes (Fig. 3b). The fibrous tissue in the defect site of rabbits in the control group was interspersed with a large number of fibroblasts and inflammatory cells at 4 weeks. At 8 and 12 weeks, the granulation tissue had become calcified, and by 24 weeks, the majority of the defect region had formed bony trabeculae and osteoid. In the upper layer, the fibrous tissue was poorly arranged and had formed transparent cartilage; the adjacent host cartilage had thinned and begun to degenerate (Fig. 3c).

\section{Immunohistochemical detection of type II collagen}

Positive type II collagen staining was found in the PVA/ $\mathrm{n}-\mathrm{HA}+\mathrm{PA} 66$ implant group at 12 weeks in the gaps between the PVA and adjacent articular cartilage and on the surface of PVA biomaterial. At 24 weeks, type II collagen staining had increased at the cartilaginous margin and on the surface of the PVA biomaterial (Fig. 4a). For rabbits in the PVA implant group, weak type II collagen staining was observed in the gap between the PVA biomaterial and the adjacent tissue, with positive staining on the surface of the PVA and in the adjacent cartilage. There was a slight increase in this staining at 24 weeks, but the staining on the surface of PVA biomaterial and in the adjacent cartilage was attenuated (Fig. 4b). In the control group, a small amount of type II collagen was present at 12 weeks, presenting as weak positive staining on the surface of the defect and in the adjacent articular cartilage. At 24 weeks, type II collagen staining was attenuated on the surface of the defect and in the adjacent articular cartilage (Fig. 4c).

\section{Scanning electron microscopy observation}

In the PVA/n-HA+PA66 implant group, a large number of fibroblasts were found in the pores of n-HA+PA66 biomaterial in the lower layer at 12 weeks, along with substantial osteoid. The osteoid was arranged in strips, and the gap between the n-HA+PA66 biomaterial and adjacent tissues contained numerous fibroblasts and collagen, with some calcified fibrous tissue surrounding the PVA in the upper layer. At 24 weeks, the n-HA+PA66 biomaterial pores in the lower layer contained osteoid that made connections with the adjacent tissue, and the PVA in the upper layer was tightly connected with the n-HA+PA66 biomaterial in the lower layer (Fig. 5a). By comparison, in the PVA implant group, a poor connection with the adjacent tissue was observed at 12 weeks,

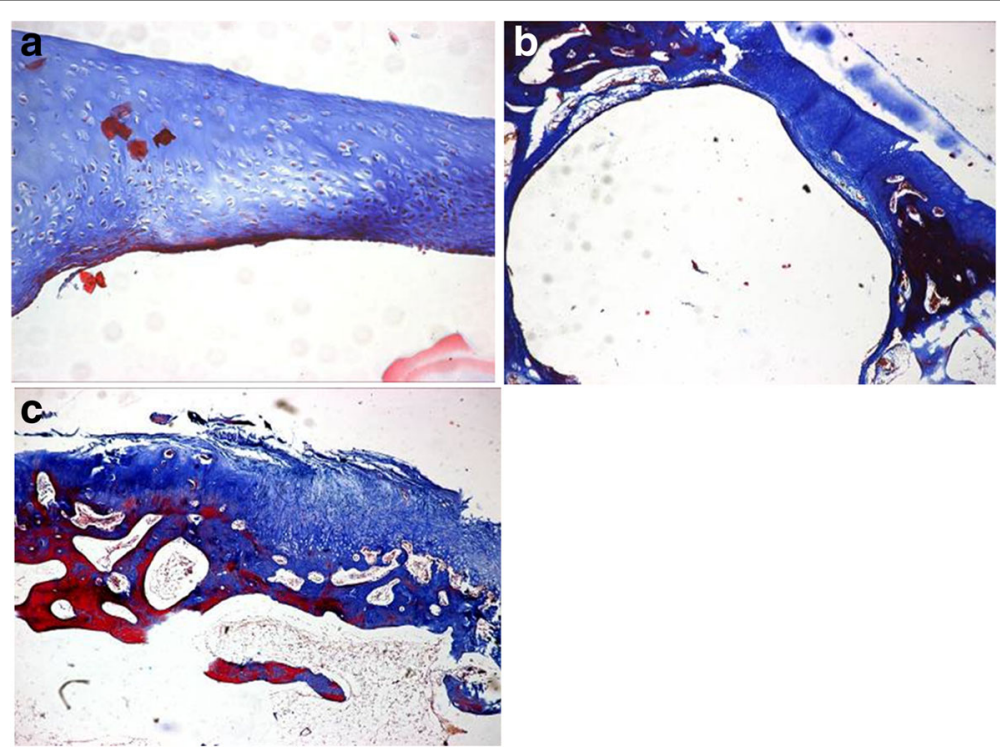

Fig. 3 At 24 weeks after implantation of polyvinyl alcohol/nano-hydroxyapatite and polyamide 66, part of the polyvinyl alcohol surface was covered by collagen $(\times 40)(\mathbf{a})$. At 24 weeks in the PVA implant group, the adjacent cartilage had thinned and continued to show degenerative changes $(\times 40)(\mathbf{b})$. At 24 weeks in the control group, the fibrous tissue was poorly arranged and had formed transparent cartilage $(\times 40)(\mathbf{c})$ 

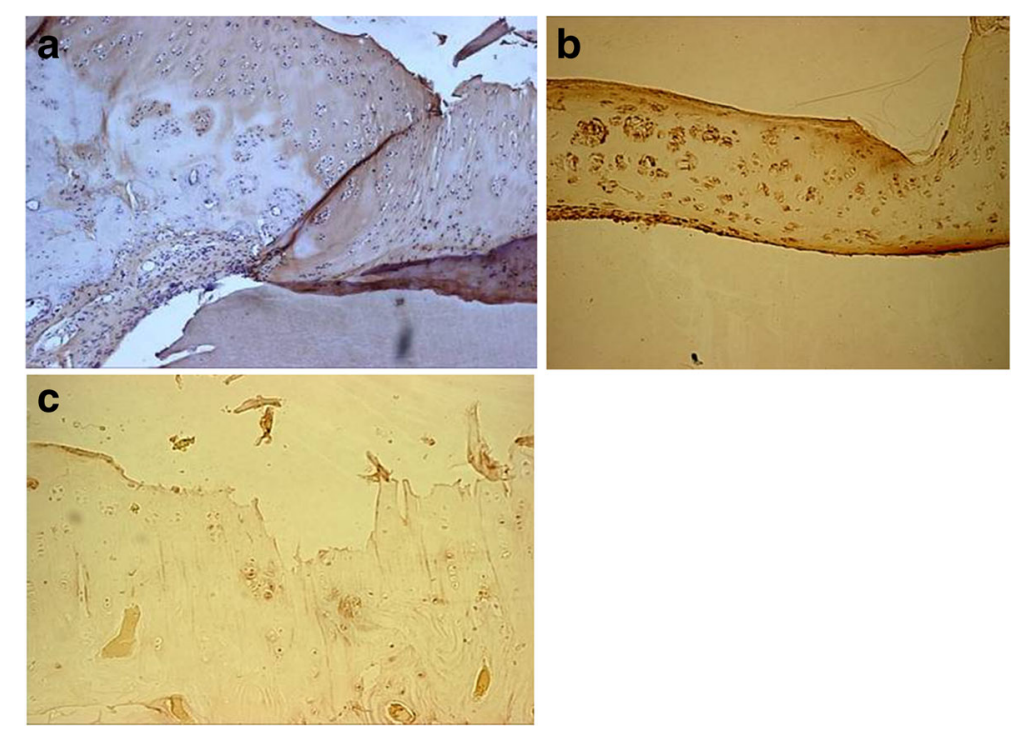

Fig. 4 At 24 weeks in PVA/n-HA+PA66 implant group, immunohistochemical detection of type Il collagen showed positive staining on the surface and in the peripheral region of the polyvinyl alcohol implant $(\times 100)(\mathbf{a})$. At 24 weeks in the PVA implant group, weak type II collagen staining was observed in the gap between the PVA biomaterial and the adjacent tissue $(\times 100)(\mathbf{b})$. At 24 weeks in the control group, a small amount of type II collagen was present, presenting as weak positive staining on the surface of the defect $(\times 100)(\mathbf{c})$

with an obvious margin. Part of the PVA in the upper layer was covered by fibrous strips. At 24 weeks, part of the adjacent sclerotin had pulled away, enlarging the gap (Fig. 5b). In the control group, the lower part of defect region was filled with solid sclerotin and the surface was covered by a layer of fibrous tissue at 12 weeks. At 24 weeks, the lower part of the defect was filled with sclerotin, and the upper part contained a disordered, scar-like fibrous tissue (Fig. 5c).

\section{Discussion}

Articular cartilage is a tissue without blood vessels, lymphatic vessels, and nerves. As such, chondrocytes
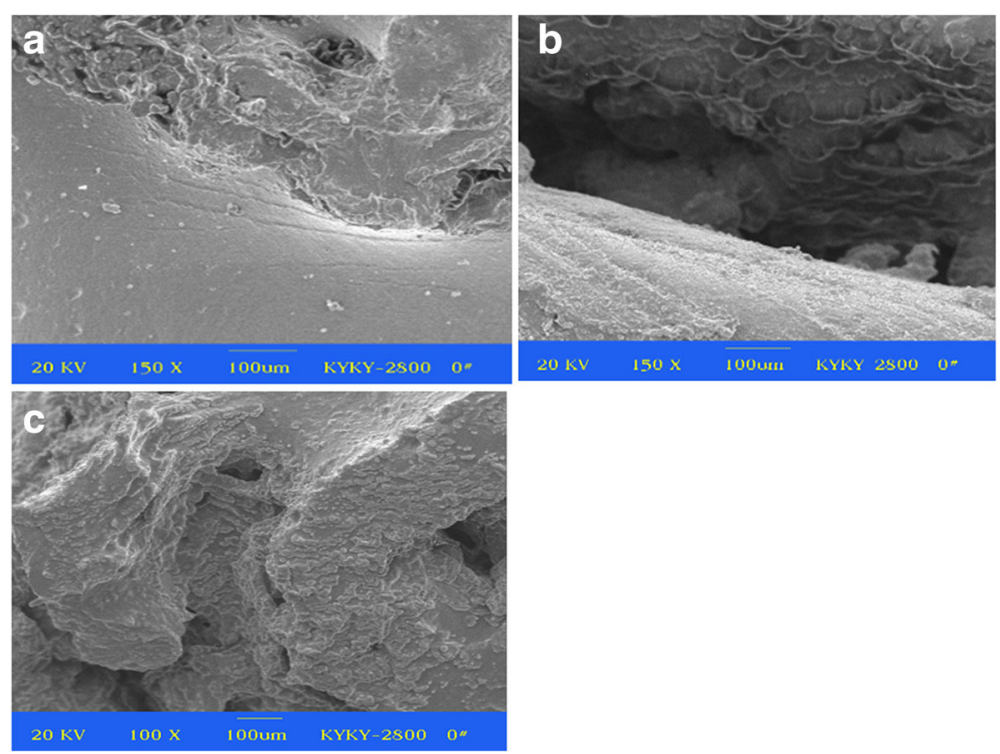

Fig. 5 At 24 weeks postoperatively, the n-HA+PA66 biomaterial in the lower layer was tightly connected with adjacent tissue and the PVA biomaterial in the upper layer was tightly connected with the n-HA+PA66 biomaterial in the lower layer (SEM $\times 150)(\mathbf{a})$. In the PVA implant group, a poor connection and gap with the adjacent tissue were observed $($ SEM $\times 150)(\mathbf{b})$. In the control group, the lower part of defect was filled with sclerotin and the upper part contained a disordered, scar-like fibrous tissue $($ SEM $\times 150)($ c) 
under general circumstances have limited capacity to undergo mitosis or regenerate the cartilage in response to damage or deterioration. According to the structure and characteristics of cartilage, a substitute material used as artificial cartilage should meet the following requirements: good biomechanical property, excellent lubricity and wear resistance, ability to induce chondrocyte growth, firm connection with bone base, and biocompatibility.

\section{Histocompatibility of composite biomaterial PVA/n-HA +PA66}

PVA hydrogel exhibits physical properties that are more similar to in vivo tissue than many other artificial composites. First, its expansive capability and water permeability contribute to its overall satisfactory biocompatibility [19]. Second, its flexibility and elasticity can reduce the load experienced by surrounding cells and tissue. Third, PVA exhibits a good biomechanical property, which is similar to the elastic modulus of cartilage, and has a small surface friction coefficient [20]. Therefore, PVA hydrogel is currently considered a good substitute biomaterial for articular cartilage [21-23].

Some scholars have performed studies to assess the cellular toxicity, safety, and excretion of PVA. Strong evidence exists that PVA does not cause hemolysis, allergic response, or skin irritations [24]. Our results demonstrate that PVA exhibited good compatibility with the adjacent articular cartilage; after the PVA implantation, the adjacent articular cartilage did not present with any degenerative changes, type II collagen was secreted, and chondrocytes were arranged in order. After 4 weeks, some chondrocytes were observed on the PVA surface and filled the gap between the PVA and the adjacent articular cartilage. At 24 weeks, the articular cartilage surrounding the PVA grew well and did not present with any obvious degenerative changes, showing positive type II collagen staining on the surface and at the biomaterial edge, suggestive of cartilage growth. These findings indicate that PVA exhibits good biocompatibility with adjacent host articular cartilage.

HA has a good osteoconductivity and has been well accepted as a bone repair substitute [25]. PA66 is a polymer with strong intensity, high flexibility, and good stability. Previous studies have shown that the combination of these two materials yields a high molecular weight polymer, n-HA+PA66, that was initially prepared under international advanced standards using Chinese intellectual property. In this study, according to human bone tissue compositions, we found that our novel biomaterial exhibited the strong rigidity of n-HA and the highly flexible nature of PA66, thereby generating a structure with similar properties to the bone and articular cartilage that was appropriate for presenting the PVA biomaterial [26-28]. Zhang et al. [29] also evaluated the biological characteristics of the n-HA/PA66 composite biomaterial in vivo and in vitro, showing that the n-HA/PA66 composite biomaterial did not dissolve in the blood and induced no cell toxicity, skin irritation or allergic response, and no pyrogen reaction or other adverse reactions after intramuscular implantation for 90 days or bony implantation for 180 days. Using this composite to repair dog mandibular cortical defects, Zheng et al. [30] found that, after surgery, the wound healed well, there were no rejections, the implant made strong connections with the bone tissue, and displayed good osteoconductivity, suggesting good biocompatibility and biological activity of the material.

\section{Integrated composite material formation by firm connection of upper and lower layers of interfaces}

The PVA/n-HA+PA66 composite consisted of a uniform crystal structure, with firm bonds to polyamide, as evidenced by electron microscopy (Fig. 1). PVA and $\mathrm{n}-\mathrm{HA}+\mathrm{PA} 66$ can be integrated by freeze-thaw cycles and casting because of the porosity of the n-HA +PA66 compound, which is suitable for permeation of liquid compositions. Part of the dissolved PVA compositions casted onto the n-HA+PA66 can directly permeate into the pores of n-HA+PA66. PVA and nHA+PA66 form a steady connection after repeated freeze-thaw cycles.

\section{Stability and advantages of integrated composite material after implantation}

Under normal conditions, subchondral and cancellous bones below the articular cartilage play an important role in protecting the articular cartilage from high stress. When the joints are exposed to high loads, the subchondral bone assigns the majority of this stress to the cancellous bone, which is arranged in a radial manner to greatly decrease the stress to articular cartilage. Brittberg et al. [31] reported that cartilage defects often involve the subchondral bone; they result in destruction of the normal cartilage and induce subsequent changes to the mechanical properties of the joint, leading to a decreased healing rate and degenerative changes. For this reason, the PVA hydrogel used as a substitute of articular cartilage should provide sufficient support to the subchondral and cancellous bones. However, a good connection between the PVA hydrogel and the bone has, until now, remained problematic. Recently, Gu et al. [32] used mechanical-chemical methods to investigate a firm connection between PVA hydrogel and metal (as a bottom layer for bone). In addition, Oka et al. [33] implanted PVA-H into the cartilage defect region and fixed it using tissue grown in titanium mesh pores. Some 
therapeutic effects were acquired, but the long-term biomechanical properties of these composites deserve further investigation.

In this study, we designed a novel artificial cartilage substitute biomaterial to solve this problem with PVA presentation for joint repair. The upper layer was made of PVA and the lower layer of n-HA+PA66, integrated by freeze-thaw cycles and casting. The upper layer functioned to substitute for articular cartilage while the lower layer substituted as a subchondral bone. The lower layer exhibited a porous structure to facilitate the ingrowth of fibrous and bony tissue. The formation of biological self-locking fixation of the n-HA+PA66 after implantation can effectively prevent displacement of the implant and greatly improve the stability and function of the artificial cartilage upper layer of the biomaterial. PVA exhibits a good stability throughout the entire lifespan, which plays a positive role in its functioning as articular cartilage.

Our results confirmed that the composite PVA/n-HA +PA66 immediately fixed into the defect, becoming integrated with the adjacent subchondral bone. At 4 weeks after implantation, the lower part of the biomaterial showed preliminary connections with the adjacent tissue. After 8 weeks, n-HA+PA66 in the lower layer attained good connections with the adjacent tissue, that gradually calcified into bone tissue. Thus, the n-HA+PA66 in the lower layer offers a strong support to the upper PVA and contributes to PVA functioning as a substitute articular cartilage.

Our results showed that the PVA alone lacked the necessary support to subchondral bone, being extremely unstable and easily deformable under the influences of external forces owing to lacking of good fixation. Electron microscopy results showed an obvious boundary between the entire PVA and the adjacent tissue that likely arose because the bone tissue and fibrous tissue cannot grow into the inner portion of the PVA. This leads to poor interconnectivity, displacement of PVA biomaterial, increased PVA abrasion, a non-uniform stress distribution, and finally degeneration of adjacent cartilage. It may even lead to osteophyma.

\section{Conclusion}

Taken together, the composite biomaterial PVA/n-HA +PA66 serves as a good integrated biomaterial substitute for articular cartilage and subchondral bone. The implanted $\mathrm{PVA} / \mathrm{n}-\mathrm{HA}+\mathrm{PA} 66$ can reconstruct the smooth cartilage surface, reduce abrasion, postpone or prevent the occurrence of osteoarthritis, and provide an alternative biomaterial for repair of articular cartilage defects. However, the problem of abrasion in long-term application of PVA biomaterial deserves further investigation.
Abbreviations

n-HA+PA66: nano-hydroxyapatite (n-HA) and polyamide 66 (PA66):

PVA: Polyvinyl alcohol hydrogel

Acknowledgments

Not applicable

Funding

No funding was received.

Availability of data and materials

All data and materials were presented in the main paper.

\section{Authors' contributions}

TG conceived and designed the experiments. TG, XT, and BL performed the experiments. TY and YL analyzed the data. TG contributed reagents/materials/ analysis tools. TG wrote the paper. All authors read and approved the final manuscript.

Ethics approval and consent to participate

The study was approved by the Ethics Committee of Guizhou Province People's Hospital. Consent to participate is not applicable.

Consent for publication

Not applicable

\section{Competing interests}

The authors declare that they have no competing interests.

\section{Publisher's Note}

Springer Nature remains neutral with regard to jurisdictional claims in published maps and institutional affiliations.

\section{Author details}

'Department of Orthopedics, Guizhou Province People's Hospital, Guiyang, Guizhou province 550002, China. ${ }^{2}$ Department of Orthopedics, West China Hospital, Sichuan University, Chengdu, Sichuan province 610041, China.

${ }^{3}$ Nanometer Analytical and Testing Center, Sichuan University, Chengdu,

Sichuan province 610041, China.

Received: 27 April 2017 Accepted: 26 October 2017

Published online: 15 November 2017

\section{References}

1. Zhang HB, Zhang BC, Zhang HJ, Zheng SM. Experimental study of hepatocyte growth factor in repairing articular cartilage defects. Zhonghua Guke Zazhi. 2000:20:181-4.

2. Yamasaki S, Mera H, Itokazu M, et al. Cartilage Repair With Autologous Bone Marrow Mesenchymal Stem Cell Transplantation: Review of Preclinical and Clinical Studies. Cartilage. 2014;5(4):196-202.

3. Shapiro F, Koide S, Glimcher MJ. Cell origin and differentiation in the repair of full-thickness defects of articular cartilage. J Bone Joint Surg Am. 1993;75(4):532-53.

4. Madsen BL, Noer HH, Carstensen JP, Nørmark F. Long-term results of periosteal transplantation in osteochondritis dissecans of the knee. Orthopedics. 2000;23(3):223-6.

5. van der Kraan PM, Buma P, van Kuppevelt T, van den Berg WB. Interaction of chondrocytes, extracellular matrix and growth factors: relevance for articular cartilage tissue engineering. Osteoarthr Cartil. 2002;10(8):631-7.

6. Wang CY, Guo XM, Fang ZQ, Duan CM, Zhao Q, Wang YH, Chen JH, Bo B, Fan M, Lu JX. Repair of sheep articular cartilage defects by tissue engineered cartilage: an experimental study. Junshi Yixue. 2002;26:81-5.

7. Li SP. Introduction of biomedical materials. Wuhan: Wuhan Gongye Daxue Chubanshe; 2000.

8. Ząbek A, Małecka B, Kołodzińska A, Maziarz A, Lelakowski J, Kutarski A. Early abrasion of outer silicone insulation after intracardiac lead friction in a patient with cardiac device-related infective endocarditis. Pacing Clin Electrophysiol. 2012;35(6):e156-8.

9. Tsui YK, Gogolewski S. Microporous biodegradable polyurethane membranes for tissue engineering. J Mater Sci Mater Med. 2009;20(8):1729-41. 
10. Buckwalter JA, Mankin HJ. Articular cartilage: tissue design and chondrocytematrix interactions. Instr Course Lect. 1998;47:477-86.

11. Chen G, Hoffman AS. Graft copolymers that exhibit temperature-induced phase transitions over a wide range of pH. Nature. 1995;373(6509):49-52.

12. Alhalafi AM. Applications of polymers in intraocular drug delivery systems. Oman J Ophthalmol. 2017;10(1):3-8.

13. Rafique A, Mahmood Zia K, Zuber M, Tabasum S, Rehman S. Chitosan functionalized poly(vinyl alcohol) for prospects biomedical and industrial applications: a review. Int J Biol Macromol. 2016;87:141-54.

14. Vellayappan MV, Balaji A, Subramanian AP, John AA, Jaganathan SK, Murugesan S, Mohandas H, Supriyanto E, Yusof M. Tangible nanocomposites with diverse properties for heart valve application. Sci Technol Adv Mater. 2015;16(3):033504.

15. Guo D, Xu K. Application of polyvinyl alcohol in biomedical engineering. Sheng Wu Yi Xue Gong Cheng Xue Za Zhi. 2005;22(3):602-5.

16. Alexandre N, Costa E, Coimbra S, Silva A, Lopes A, Rodrigues M, Santos M, Maurício AC, Santos JD, Luís AL. In vitro and in vivo evaluation of blood coagulation activation of polyvinyl alcohol hydrogel plus dextran-based vascular grafts. J Biomed Mater Res A. 2015;103(4):1366-79.

17. Kobayashi M, Oka M. Composite device for attachment of polyvinyl alcoholhydrogel to underlying bone. Artif Organs. 2004;28(8):734-8.

18. Ushio K, Oka M, Hyon SH, Hayami T, Yura S, Matsumura K, Toguchida J, Nakamura T. Attachment of artificial cartilage to underlying bone. J Biomed Mater Res B Appl Biomater. 2004;68(1):59-68.

19. $\mathrm{Xu} F \mathrm{FL}$, Li YB, Li JD, Mu YH. Swelling properties of nano-hydroxyaptite/ polyvinyl alcohol hydrogel composite. Cailiao Gongcheng. 2005;50:15-18,22.

20. Williams S. Mechanical testing of a new biomaterial for potential use as a vascular graft and articular cartilage replacement [dissertation]. Atlanta: Georgia Institute of Technology; 2006.

21. Katta JK, Marcolongo MS, Lowman AM, Mansmann KA. Friction and wear characteristics of PVA/PVP hydrogels as synthetic articular cartilage. IEEE. 2004:4:142-3.

22. Li Y, Gao J, Liu G, Gu Z, Ma Y, Xue H. Swelling characterization of poly (vinyl alcohol) hydrogel for prosthetic intervertebral disc nucleus. Sheng Wu Yi Xue Gong Cheng Xue Za Zhi. 2005;22(5):995-8.

23. Kobayashi M, Toguchida J, Oka M. Development of an artificial meniscus using polyvinyl alcohol-hydrogel for early return to, and continuance of, athletic life in sportspersons with severe meniscus injury. II: animal experiments. Knee. 2003;10:53.

24. Baker MI, Walsh SP, Schwartz Z, Boyan BD. A review of polyvinyl alcohol and its uses in cartilage and orthopedic applications. J Biomed Mater Res B Appl Biomater. 2012;100:1451-7.

25. Du C, Cui FZ, Feng QL, Zhu XD, de Groot K. Tissue response to nanohydroxyapatite/collagen composite implants in marrow cavity. J Biomed Mater Res. 1998:42:540-8.

26. Xu YH, Li SQ, Li ZA. Development of nanometer hydroxyapatite complex materials-cytotoxicity test of nano-hydroxyapatite by MTT-assay. Shiyong Kouqiang Yixue Zazhi. 2004;20:147-50.

27. Yang $X$, Song $Y$, Liu L, et al. Anterior reconstruction with nanohydroxyapatite/polyamide-66 cage after thoracic and lumbar corpectomy. Orthopedics. 2012;35(1):e66-73.

28. Meng $C Y$, An H, Jiang DM, Li YB, Wei J. Biocompatibility and security of new type nano hydroapatite crystals and polyamide for bone reconstruction and repair after implanting in vivo. Zhongguo Linchuang Kangfu. 2004:8:6330-545.

29. Zhang L, Li YB, Wang XJ, Wei J, Peng XL. Studies on porous scaffold made of the nano-HA/PA66 composite. J Mater Sci. 2005;40:107-10.

30. Zheng Q, Zhou LW, Wei SC, Li YB, Wei J. Experimental study on the reconstruction of mandibular defects with a new bioactive artificial bone nano-hydroxyapatite/polyamide-66 in dogs. Zhonghua Kou Qiang Yi Xue Za Zhi. 2004;39(1):60-2.

31. Brittberg M, Nilsson A, Lindahl A, Ohlsson C, Peterson L. Rabbit articular cartilage defects treated with autologous cultured chondrocytes. Clin Orthop Relat Res. 1996;326:270-83.

32. Gu ZQ, Xiao JM, Lou SQ. The mechanical-chemical attachment between the artificial articular cartilage (PVA-hydrogel) and metal substrate (or underlying bone). Biomed Mater Eng. 1999;9(5-6):347-51.

33. Oka M. Biomechanics and repair of articular cartilage. J Orthop Sci. 2001;6(5):448-56.

\section{Submit your next manuscript to BioMed Central and we will help you at every step:}

- We accept pre-submission inquiries

- Our selector tool helps you to find the most relevant journal

- We provide round the clock customer support

- Convenient online submission

- Thorough peer review

- Inclusion in PubMed and all major indexing services

- Maximum visibility for your research

Submit your manuscript at www.biomedcentral.com/submit
C Biomed Central 